ene

\title{
LOS ESTILOS DE APRENDIZAJE DE LOS ESTUDIANTES DEL GRADO EN ENFERMERÍA DE LA UNIVERSIDAD DE LA LAGUNA
}

\section{LEARNING STYLES IN NURSING STUDENTS OF THE UNIVERSITY OF LA LAGUNA}

Mª Mercedes Arias Hernández, Miriam C. González Afonso y Luis Feliciano García.

Escuela de Doctorado y Estudios de Posgrado. Universidad de La Laguna (España)

Arias Hernández, M., González Afonso, M., \& Feliciano García, L. (2020). LOS ESTILOS DE APRENDIZAJE DE LOS ESTUDIANTES DEL GRADO EN ENFERMERÍA DE LA UNIVERSIDAD DE LA LAGUNA. Revista Ene de Enfermería, 14(2).

Consultado de http://www.ene-enfermeria.org/ojs/index.php/EnE/article/view/ 1017 


\section{Resumen}

Los estilos de aprendizaje, entendidos como la tendencia a desarrollar unas preferencias en las estrategias para aprender, constituyen una variable influyente en el desarrollo de las nuevas competencias del estudiante de enfermería dentro del nuevo modelo de formación universitaria, centrado en el estudiante como motor de su proceso de aprendizaje.

Se realizó un estudio descriptivo de corte transversal entre los estudiantes de Enfermería de la Universidad de La Laguna, con el objetivo de identificar sus estilos de aprendizaje preferentes mediante el Cuestionario Honey-Alonso de Estilos de Aprendizaje (CHAEA). Los resultados obtenidos concluyen que nuestros estudiantes presentan un perfil de aprendizaje con predominio de los estilos reflexivo y teórico.

Palabras clave: Enfermería; Estudiantes; Educación; Estilos de aprendizaje;

\section{Abstract}

Learning styles are mental processes carried out by students to process, understand and adopt the information during the education process. The objective is to describe the learning style of students in the Nursing Degree, University of La Laguna. This is a transversal study with 303 students from all levels. The Honey-Alonso Learning Styles Questionnaire (CHAEA), validated in Spanish context was used. The results conclude that our students present a reflexive and theoretic learning style.

Key Words: Nursing; Students, Education; Learning styles 


\section{INTRODUCCIÓN}

La integración de los estudios de Enfermería en el Espacio Europeo de Educación Superior (EEES) ha supuesto una oportunidad para la mejora de la calidad de la formación enfermera que, se ajusta al pensamiento del paradigma sociocrítico en el que se enmarca la Enfermería española actual. La práctica reflexiva y el pensamiento crítico permiten la emancipación y, por extensión, la autonomía del paciente por la vía de la participación activa en la solución de sus problemas de salud. Este proceso se origina con la toma de conciencia del papel que deben desempeñar el profesional y el ciudadano/paciente en la planificación de los cuidados de salud. (1)

El nuevo modelo de formación universitaria enfatiza la enseñanza basada en competencias y, la responsabilidad del estudiante sobre su proceso de aprendizaje. El estilo de aprendizaje, entendido como la tendencia a desarrollar unas preferencias globales en la elección de las estrategias para aprender, constituye una variable que influye en esta competencia.

Durante el curso académico 2017-18 hemos realizado una investigación descriptiva de corte transversal, entre los estudiantes de Enfermería de la Universidad de La Laguna, con el objetivo de identificar sus estilos de aprendiza- je predominantes que permita replantear la planificación docente y las metodologías de enseñanza y aprendizaje.

El paradigma actual de la enseñanza y la formación superior está centrado en el estudiante que aprende. Este paradigma se basa en el desarrollo del conocimiento y el aprendizaje a lo largo de la vida. El estilo de aprendizaje, entendido como la tendencia a desarrollar unas preferencias globales en la elección de estrategias para aprender, constituye una variable que influye en la capacidad de aprender. El conocimiento del estilo propio de aprendizaje, es uno de los factores claves para desarrollar la capacidad de aprender a aprender. Los estilos de aprendizaje pueden ser modificados, siendo una responsabilidad de los docentes ayudar al estudiante a descubrir su estilo y aprender a adaptarlo a las experiencias de cada situación. (2)

Nuestra posición teórica respecto al tema de los estilos de aprendizaje se sitúa en la propuesta de Alonso, Gallego y Honey (3) y el estilo de aprendizaje de Honey y Mumford (4) basada en la teoría de Kolb (5) que define cuatro tipos de estilos de aprendizaje de acuerdo a la forma de organizar y trabajar que son estilos activos, teóricos, reflexivos y pragmáticos. Castro y Guzmán (6) identifican las siguientes características definitorias para cada estilo de aprendizaje (Tabla 1): 
Tabla 1. Características principales de los distintos tipos de aprendizaje, según el modelo de Kolh

\begin{tabular}{|c|c|c|}
\hline Estilo & Descripción & $\begin{array}{l}\text { Características } \\
\text { principales }\end{array}$ \\
\hline ACTIVO & $\begin{array}{l}\text { Busca experiencias nuevas, son de mente abierta, nada } \\
\text { escépticos y acometen con entusiasmo las tareas nuevas. } \\
\text { Piensan que hay que intentarlo todo, por lo menos una vez. En } \\
\text { cuanto desciende la excitación de una novedad comienzan a } \\
\text { buscar la próxima. } \\
\text { Se crecen ante los retos que suponen las experiencias nuevas y } \\
\text { se aburren con los plazos largos. Son personas de grupo que se } \\
\text { involucran en los asuntos de los demás y centran a su alrededor } \\
\text { todas las actividades }\end{array}$ & $\begin{array}{l}\text { Animador } \\
\text { Improvisador } \\
\text { Descubridor } \\
\text { Arriesgado } \\
\text { Espontáneo }\end{array}$ \\
\hline REFLEXIVO & $\begin{array}{l}\text { Antepone la reflexión a la acción y observa con detenimiento las } \\
\text { distintas experiencias. Les gusta considerar las experiencias y } \\
\text { observarlas desde diferentes perspectivas. } \\
\text { Recogen datos analizándolos con detenimiento antes de llegar } \\
\text { a una conclusión. Son prudentes, les gusta considerar todas las } \\
\text { alternativas posibles antes de realizar un movimiento. Disfrutan } \\
\text { observando la actuación de los demás, escuchan a los demás y } \\
\text { no intervienen hasta que no se han adueñado de la situación. } \\
\text { Crean a su alrededor un aire ligeramente distante y } \\
\text { condescendiente }\end{array}$ & $\begin{array}{l}\text { Ponderado } \\
\text { Concienzudo } \\
\text { Receptivo } \\
\text { Analítico } \\
\text { Exhaustivo }\end{array}$ \\
\hline TEÓRICO & $\begin{array}{l}\text { Da un enfoque lógico de los problemas, necesitan integrar la } \\
\text { experiencia en un marco teórico de referencia. Enfocan los } \\
\text { problemas de forma vertical, escalonada por etapas lógicas. } \\
\text { Tienden a ser perfeccionistas. Integran los hechos en teorías } \\
\text { coherentes. Les gusta analizar y sintetizar. Son profundos en su } \\
\text { sistema de pensamiento a la hora de establecer principio, } \\
\text { teorías y modelos. Para ellos, si es lógico es bueno. Buscan la } \\
\text { racionalidad y la objetividad huyendo de lo subjetivo y lo } \\
\text { ambiguo }\end{array}$ & $\begin{array}{l}\text { Metódico } \\
\text { Lógico } \\
\text { Objetivo } \\
\text { Crítico } \\
\text { Estructurado }\end{array}$ \\
\hline PRAGMÁTICO & $\begin{array}{l}\text { Su punto fuerte es la experimentación y la aplicación de ideas. } \\
\text { Descubren el aspecto positivo de las nuevas ideas y } \\
\text { aprovechan la primera oportunidad para experimentarlas. Les } \\
\text { gusta actuar rápidamente y con seguridad con aquellas ideas y } \\
\text { proyectos que les atraen. Tienden a ser impacientes cuando hay } \\
\text { personas que teorizan. Pisan la tierra cuando hay que tomar } \\
\text { una decisión o resolver un problema. Su filosofía es "siempre se } \\
\text { puede hacer mejor; si funciona es bueno" }\end{array}$ & $\begin{array}{l}\text { Experimentador } \\
\text { Práctico } \\
\text { Directo } \\
\text { Eficaz } \\
\text { Realista }\end{array}$ \\
\hline
\end{tabular}

- estilo activo: indica una preferencia por implicarse en nuevas experiencias e involucrarse en el trabajo en equipo. Suelen ser personas espontáneas, creativas, innovadoras, deseosas de aprender y resolver problemas.
- estilo reflexivo: consideran las experiencias y las observan desde diferentes perspectivas. Reúnen datos y los analizan con detenimiento antes de llegar a alguna conclusión. Son prudentes, observadores y consideran todas las al- 
ternativas posibles antes de realizar un movimiento.

- estilo teórico: adaptan e integran las observaciones dentro de las teorías lógicas y complejas. Son profundos en su sistema de pensamiento, metódicos y estructurados. Buscan la racionalidad y la objetividad huyendo de lo subjetivo.

- estilo pragmático: prefieren la aplicación de las ideas. Son experimentadores, prácticos, directos y realistas.

Los estilos de aprendizaje se definen como los rasgos cognitivos, afectivos y fisiológicos que sirven como indicadores relativamente estables, de cómo las personas perciben, interaccionan $y$ responden a sus ambientes de aprendizaje.(7)

Las manifestaciones externas acerca del aprendizaje responden, por una parte a disposiciones naturales de cada individuo $y$, por otra a los resultados de experiencias y aprendizajes pasados, diferentes según los contextos y las culturas. La Teoría de los Estilos de Aprendizaje confirma esta diversidad entre los individuos $y$, propone un camino para mejorar por medio de la reflexión personal y de las peculiaridades en el modo de aprender. (8)

David Kolb propone un modelo de aprendizaje que depende del modo de procesar la información y de la experiencia y reflexiona en profundidad sobre las repercusiones de los estilos de aprendizaje en la vida adulta de las personas. Según Kolb, cada sujeto enfoca el aprendizaje de una forma peculiar producto de la herencia, las experiencias anteriores y las exigencias actuales del ambiente en el que se mueve. (5)

Alonso, Gallego y Honey (3) destacan que la intervención educativa debe tener como objetivo prioritario posibilitar que los estudiantes logren aprendizajes significativos por sí solos, es decir, que sean capaces de aprender a aprender y plantea que, una de las formas de alcanzar este objetivo, nace del conocimiento del propio estilo de aprendizaje.

Las investigaciones cognitivas han demostrado que no existe una sola forma de aprender debido al modo de relacionarse las personas con el mundo y la intención que tenga cada uno frente a lo que quiere aprender. Esta realidad lleva a que cada persona tienda a desarrollar ciertas preferencias o disposiciones generales definiendo los estilos de aprendizaje, entendiéndose como rasgos cognitivos, afectivos y fisiológicos. Los individuos no tienen un estilo fijo, tienen un perfil con ciertas predominancias, y éstas se acentuarán de acuerdo con las situaciones del entorno. La comprensión del propio estilo constituye uno de los factores claves para 
desarrollar la capacidad de aprender a aprender. (9)

En España, se realiza la primera investigación sobre estilos de aprendizaje en una muestra de 89 estudiantes de enfermería utilizando el cuestionario Honey-Alonso (CHAEA), como instrumento de medida validado en este estudio. (2)

La integración de los estudios de Enfermería en el Espacio Europeo de Educación Superior (EEES) en el año 2009, ha supuesto una oportunidad para la mejora de la calidad de la enseñanza superior en la formación enfermera. Esta condición se ajusta al pensamiento del paradigma sociocrítico, en el que se enmarca la Enfermería actual española, que ha evolucionado de la racionalidad técnica a la práctica reflexiva. La práctica reflexiva y el pensamiento crítico permiten la emancipación y, por extensión, la autonomía del paciente por la vía de la participación activa en la solución de sus problemas de salud. Este proceso se origina con la toma de conciencia del papel que deben desempeñar el profesional y el ciudadano/paciente en la planificación de los cuidados de salud. (1)

La sociedad del siglo XXI demanda personas y profesionales que, a través de un aprendizaje continuo, se conviertan en sujetos autónomos, capaces de tomar conciencia de sus propios procesos mentales al enfrentarse con los problemas, analizarlos adecuadamente, planificar, supervisar y evaluar la propia actuación.

\section{OBJetivos de LA INVESTIGACIÓN}

En el nuevo contexto del Espacio Europeo de Educación Superior (EEES) se hace necesario analizar el perfil de aprendizaje del colectivo de estudiantes de enfermería, ya que es un indicador útil para conocer sus preferencias por aprender de diversas formas.

Con este trabajo de investigación queremos analizar el perfil de aprendizaje de las/los estudiantes del Grado en Enfermería de la Universidad de La Laguna.

Establecemos como objetivos específicos de nuestra investigación:

Identificar los estilos de aprendizaje predominantes de los estudiantes de Enfermería durante el curso académico 2017-2018.

Determinar la relación entre el estilo de aprendizaje predominante y las variables vía de acceso a la universidad, experiencia profesional y grupo. 


\section{MATERIAL Y MÉTOdO}

Se ha realizado un estudio descriptivo de corte transversal, mediante la recogida de datos a partir de cuestionarios on-line, entre las/os estudiantes de todos los niveles en la titulación de Grado en Enfermería de la Universidad de La Laguna (España).

Durante el curso académico 2017-18 se matricularon en la titulación de Grado Enfermería un total de 576 estudiantes, distribuidos entre el Grupo La Laguna (406) y, Grupo La Palma (170). La selección de la muestra se realiza mediante un muestreo probabilístico aleatorio simple. Participaron en nuestra investigación 303 estudiantes de todos los niveles.

Las variables seleccionadas para el estudio incluyen el estilo de aprendizaje, como variable principal y, como variables secundarias se registraron variables sociodemográficas (edad, género) y académicas (grupo, curso, vía de acceso, dedicación y experiencia profesional).

El instrumento utilizado en nuestro estudio es el Cuestionario HoneyAlonso de Estilos de Aprendizaje (CHAEA), validado en contexto español. El cuestionario consta de 80 ítems de respuesta dicotómica, de los cuales 20 se corresponden con cada estilo de aprendizaje y distribuidos aleatoriamente. La puntuación absoluta que cada su- jeto de estudio obtiene en cada grupo de 20 ítems, indica el nivel que alcanza en cada uno de los estilos de aprendizaje. Los resultados obtenidos valoran las preferencias de cada estudiante en cada uno de los estilos, lo que nos permite obtener los perfiles predominantes de aprendizaje de este grupo. (10)

La recogida de datos se realizó por encuestación on-line entre los meses de octubre de 2017 y marzo de 2018. El cuestionario se realizó de manera anónima y voluntaria, mediante mensaje de correo electrónico donde se explicaba la finalidad del estudio, se solicitaba el consentimiento de participación en el mismo $y$, se daban las instrucciones para cumplimentar los formularios.

El procesamiento y análisis de los datos se realizó con el programa estadístico informático Statistical Package for the Social Sciences (SPSS), en su versión 23 en español, y se aplicaron estadísticos descriptivos: de frecuencia absoluta y relativa, medias, desviaciones típicas (DT) y correlaciones.

Aspectos éticos de la investigación

Esta investigación se ajusta a la Ley Orgánica 3/2018, de 5 de diciembre, de Protección de Datos Personales y garantía de los derechos digitales. Se ha informado a los sujetos de estudio de la 

LOS ESTILOS DE APRENDIZAJE DE LOS ESTUDIANTES DEL GRADO EN ENFERMERÍA DE LA UNIVERSIDAD DE LA LAGUNA

naturaleza del estudio, del carácter voluntario de la participación y que los datos obtenidos serán usados de forma confidencial, en cumplimiento del Artículo 6.7 del Reglamento de Régimen Interior de la Escuela de Doctorado y Estudios de Posgrado de la Universidad de La Laguna.

Conflictos de intereses: ninguno Financiación: ninguna

\section{RESULTADOS}

Descripción de la muestra

Se cumplimentaron 303 cuestionarios que, representa un índice de participación del $52 \%$ de la población. De estos corresponden $66(21,8 \%)$ a primer curso; $96(31,7 \%)$ a segundo curso; 54 $(17,8 \%)$ a tercer curso y, $87(28,7 \%)$ a cuarto curso. El alumnado del Grupo de La Laguna participó en mayor proporción (71,9\%) que el Grupo de La Palma $(28,1 \%)$ (Tabla 2).

\begin{tabular}{|l|l|l|}
\hline $\begin{array}{l}\text { Tabla 2. Distribución de la muestra por cursos, en } \\
\text { frecuencias absolutas y relativas }\end{array}$ \\
\hline Curso & Frecuencia & Porcentaje (\%) \\
\hline Primero & 66 & 21,8 \\
\hline Segundo & 96 & 31,7 \\
\hline Tercero & 54 & 17,8 \\
\hline Cuarto & 87 & 28,7 \\
\hline TOTAL & 303 & 100,0 \\
\hline
\end{tabular}

La mayoría de los participantes en el estudio son mujeres $(80,2 \%)$ con una edad media de 22 años (DT 6,13); el $85,5 \%$ ha accedido a la titulación por la vía de la prueba de acceso a la universidad y, el $89,8 \%$ se encuentra en modalidad de dedicación completa a sus estudios. El $80,2 \%$ de las/os estudiantes manifestaron que no tienen experiencia laboral, frente a un $18,1 \%$ que sí la tiene.

Análisis descriptivo de los estilos de aprendizaje

El perfil de aprendizaje predominante entre los estudiantes de Enfermería durante el curso 2017-18, en los grupos de La Laguna y La Palma, es el estilo reflexivo seguido del estilo teórico (Tabla 3). 

LOS ESTILOS DE APRENDIZAJE DE LOS ESTUDIANTES DEL GRADO EN ENFERMERÍA DE LA UNIVERSIDAD DE LA LAGUNA

Tabla 3. Comparación del perfil de aprendizaje de los estudiantes del Grado en Enfermería de la Universidad de La Laguna, según curso y grupo

Grupo La Laguna

\begin{tabular}{|l|l|l|l|l|l|l|l|l|l|}
\hline Estilos de aprendizaje & \multicolumn{2}{l}{ Primer curso } & \multicolumn{2}{l|}{ Segundo curso } & \multicolumn{2}{l|}{ Tercer curso } & \multicolumn{2}{l|}{ Cuarto curso } \\
\hline & Media & DT & Media & DT & Media & DT & Media & DT \\
\hline Estilo pragmático & 12,94 & 2,90 & 12,93 & 2,40 & 12,45 & 2,24 & 12,76 & 2,37 \\
\hline Estilo teórico & 13,92 & 2,88 & 14,10 & 2,61 & 14,61 & 2,16 & 14,68 & 2,38 \\
\hline Estilo activo & 11,18 & 3,42 & 10,32 & 3,10 & 9,21 & 2,67 & 10,02 & 3,28 \\
\hline Estilo reflexivo & 15,90 & 2,76 & 15,97 & 2,48 & 16,91 & 1,91 & 16,47 & 2,32 \\
\hline
\end{tabular}

Grupo La Palma

\begin{tabular}{|l|l|l|l|l|l|l|l|l|l|}
\hline Estilos de aprendizaje & Primer curso & \multicolumn{2}{l|}{ Segundo curso } & \multicolumn{2}{l|}{ Tercer curso } & \multicolumn{2}{l|}{ Cuarto curso } \\
\hline & Media & DT & Media & DT & Media & DT & Media & DT \\
\hline Estilo pragmático & 11,53 & 2,10 & 13,56 & 3,60 & 12,35 & 2,83 & 12,14 & 2,33 \\
\hline Estilo teórico & 13,76 & 2,73 & 14,96 & 2,85 & 13,70 & 2,75 & 14,38 & 2,77 \\
\hline Estilo activo & 9,41 & 4,12 & 11,33 & 2,99 & 11,00 & 3,29 & 9,33 & 3,41 \\
\hline Estilo reflexivo & 15,59 & 2,37 & 16,85 & 2,64 & 15,65 & 2,70 & 16,00 & 2,81 \\
\hline
\end{tabular}

Al analizar por cursos, se observa que los estudiantes de cuarto muestran la puntuación más alta en el estilo reflexivo. El grupo de La Laguna obtiene una media de 16,47 y el de La Palma de 16,00 puntos.

El estilo de aprendizaje activo, es el de menor preferencia entre las/los estudiantes de ambos grupos del Grado de Enfermería de la Universidad de La Laguna, durante el curso 2017-18.

El alumnado de los cursos avanzados (tercero y cuarto) del grupo de La Laguna manifiestan un estilo de aprendizaje reflexivo más marcado que las/os estudiantes de primero y segundo curso.
Esta tendencia no se muestra igual en el grupo de La Palma.

Con el fin de estudiar la existencia de diferencias significativas entre los estilos de aprendizaje de los estudiantes se realizó en primer lugar un test de homogeneidad donde no se observaron diferencias significativas en los estilos de aprendizaje de los estudiantes, según el nivel de estudio, puesto que ninguno de los niveles de significancia supera el 0,01 . Posteriormente, se llevó a cabo una prueba ANOVA con los diferentes estilos por grupos para contrastar estos resultados que confirma la no existencia de diferencias significativas entre los es- 

LOS ESTILOS DE ARRENDIZAJE DE LOS ESTUDIANTES DEL GRADO EN ENFERMERÍA DE LA UNIVERSIDAD DE LA LAGUNA

tilos de aprendizaje de los estudiantes

del Grado en Enfermería (Tabla 4).

\begin{tabular}{|c|c|c|c|c|c|c|}
\hline \multicolumn{7}{|l|}{ ANOVA } \\
\hline & & $\begin{array}{l}\text { Sum a } \quad \mathrm{de} \\
\text { cuadrados }\end{array}$ & $d f$ & $\begin{array}{l}\text { Cuadrado } \\
\text { Medio }\end{array}$ & $\mathrm{F}$ & Significación \\
\hline \multirow[t]{3}{*}{$\begin{array}{l}\text { Estilo } \\
\text { Pragmático }\end{array}$} & Intergrupos & 5,094 & 1 & 5,094 & $\begin{array}{l}0,74 \\
8\end{array}$ & 0,388 \\
\hline & Intragrupos & 2043,094 & 300 & 6,810 & & \\
\hline & Total & 2048,189 & 301 & & & \\
\hline \multirow[t]{3}{*}{$\begin{array}{l}\text { Estilo } \\
\text { Teórico }\end{array}$} & Intergrupos & 0,059 & 1 & 0,059 & $\begin{array}{l}0,00 \\
9\end{array}$ & 0,926 \\
\hline & Intragrupos & 2053,915 & 300 & 6,846 & & \\
\hline & Total & 2053,974 & 301 & & & \\
\hline \multirow[t]{3}{*}{$\begin{array}{l}\text { Estilo } \\
\text { Activo }\end{array}$} & Intergrupos & 0,924 & 1 & 0,924 & $\begin{array}{l}0,08 \\
6\end{array}$ & 0,770 \\
\hline & Intragrupos & 3239,013 & 300 & 10,797 & & \\
\hline & Total & 3239,937 & 301 & & & \\
\hline \multirow[t]{3}{*}{$\begin{array}{l}\text { Estilo } \\
\text { Reflexivo }\end{array}$} & Intergrupos & 1,248 & 1 & 1,248 & $\begin{array}{l}0,20 \\
0\end{array}$ & 0,655 \\
\hline & Intragrupos & 1868,609 & 300 & 6,229 & & \\
\hline & Total & 1869,858 & 301 & & & \\
\hline
\end{tabular}

Relación de los estilos de aprendizaje con otras variables

Al valorar la relación entre los estilos de aprendizaje y las variables de vía de acceso a la universidad (EBAU, Mayores de 25-40-45 años), experiencia en el mundo laboral y grupo de origen (La Laguna, La Palma), no se observaron diferencias significativas en la puntuación de los estilos según las variables estudiadas.

\section{Discusión}

Los hallazgos principales de este estudio aportan las siguientes conclusiones:

* Las/los estudiantes de Enfermería presentan un perfil de aprendizaje con predominio de los estilos reflexivos y teóricos, de forma general a lo largo de los cuatro cursos. Nuestros resultados coinciden con los estudios de Alonso (1992) y Canalejas (2005). Nos planteamos si, tal y como afirma Alonso, citado 

LOS ESTITOS DE APRENDIZAJE DE IOS ESTUDTANTES DEL GRADO EN ENFERMERIA DE IA UNTVERSTDAD DE LA IAGUNA

por Canalejas, las/los estudiantes de enfermería presentan un perfil de aprendizaje en el que se diferencian sus preferencias en la forma de aprender. (9)

* Se ha observado como las puntuaciones de los estilos reflexivo y teórico aumentan conforme avanzan los cursos. Como línea futura se podría plantear el estudio de la relación existente entre las metodologías docentes empleadas y la estimulación de ambos estilos entre los estudiantes.

* El estilo de aprendizaje activo y el estilo pragmático son los de menor preferencia entre los estudiantes de Enfermería. Nos planteamos la necesidad de planificar metodologías docentes que impulsen el desarrollo de ambos estilos, para que el alumnado tenga más facilidad de aprender de cualquier forma $y$, aprovechar aquellas oportunidades de aprendizaje que se presenten a lo largo de su vida. Por ejemplo, el abordaje de las variadas situaciones de estrés a las que se expondrán las-os egresadas-os que experimentarán en breve en el mundo laboral: momentos de gran urgencia, estar en contacto con el sufrimiento, dolor, invalidez e, incluso, la muerte; la interrelación con otros profesionales etc. Los estudios de Simarro (2010) concluyen que existe una relación de mayor estrés cuando los profesionales tienen niveles bajos de estilo de aprendizaje activo y cuando tienen niveles altos de los estilos de aprendizaje reflexivo y teórico. El no actuar de forma arriesgada, ni improvisada, sino con reflexión y consideración del riesgo supone también una mayor percepción de estrés ante las situaciones complicadas. (11)

También sería de interés llevar a cabo este mismo estudio con el objetivo de determinar la relación de los estilos de aprendizaje y género de los estudiantes. A lo largo de este estudio se trató de plantear este objetivo y se obtuvieron resultados que podrían servir como primera aproximación. Sin embargo, estos resultados no se han publicado debido a que no se trata de una muestra significativa, ya que hay una clara predominancia de estudiantes de género femenino (243 mujeres) frente al masculino (60 hombres). Como línea futura sería interesante ampliar la muestra masculina para poder obtener conclusiones representativas.

Por último, cabe resaltar que esta investigación no permite valorar si los estilos de aprendizaje se modifican a lo largo de la formación, por lo que se hace necesario llevar a cabo un estudio longitudinal que analice el comportamiento de un mismo grupo de estudiantes a lo largo de su formación, con la intención de investigar si el estilo de aprendizaje del 

LOS ESTILOS DE APRENDIZAJE DE LOS ESTUDIANTES DEL GRADO EN ENEERMERÍA DE LA UNIVERSIDAD DE LA LAGUNA alumnado es modificado a razón del estilo de enseñanza de los docentes. 


\section{BiBLIOGRAFÍA}

1. Moreno I.M. y Siles J. (2014). "Pensamiento crítico en enfermería: de la racionalidad técnica a la práctica reflexiva" en Aquichan, 14 (4).Edit. Universidad de La Sabana, Colombia, páginas 594-604.

2. Alonso, C. (1992). Análisis y Diagnóstico de los Estilos de Aprendizaje en Estudiantes Universitarios. Tomo II. Editorial de la Universidad Complutense. Colección Tesis Doctorales. Madrid

3. Alonso, C. M., Gallego, D. J. y Honey, P. (2012). Los estilos de aprendizaje. Procedimientos de diagnóstico y mejora. Ediciones Mensajero. 8a edic. Bilbao, España

4. Honey, O., y Mumford, A. (1986). The manual of learning styles. Editorial: Peter Honey Maidenhead

5. Kolb, D. (1984). Experiential learning: Experience as the source of learning and development. Editorial Prentice-Hall. Nueva Jersey

6. Castro, S. y Guzmán, B. (2005). "Los estilos de aprendizaje en la enseñanza y el aprendizaje: una propuesta para su implementación" en Revista de Investigación, 58, Editores: Universidad Pedagógi- ca Experimental Libertador: Instituto Pedagógico de Caracas, Venezuela, páginas 83- 102.

7. Keefe, J. (1988). Profiling and Utilizing Learning Style, NASSP Learning Style Series. Reston, Virginia

8. Massimino, L. (2006). Preferencias de estilos de aprendizaje en estudiantes universitarios de Historia de España, Italia y Alemania. Tesina. Universidad Nacional de Educación a Distancia UNED, Madrid

9. Canalejas Pérez, M.C., Martínez Martín, M., Pineda Ginés, M.C., Vera Cortés, M.L., Soto González, M., Martín Marino, A. \& Cid Galán, M. L. (2005). "Estilos de aprendizaje en los estudiantes de enfermería" en Educación Médica, 8(2), Editorial Fundación Educación médica, Barcelona, España, páginas 33-40.

10.Alonso, C. M., Gallego, D. J. y Honey, P. (2012). CHAEA. Cuestionario Honey-Alonso de Estilos de Aprendizaje. Ediciones Mensajero, Bilbao, España

11.Simarro Blasco JA et al. "Estrés en la Unidad de Cuidados Intensivos en función de los grados de estilos de aprendizaje" en Metas de Enferm, 12(10), Editorial DAE-Grupo Paradigma, Valencia, España, páginas 62-69. 